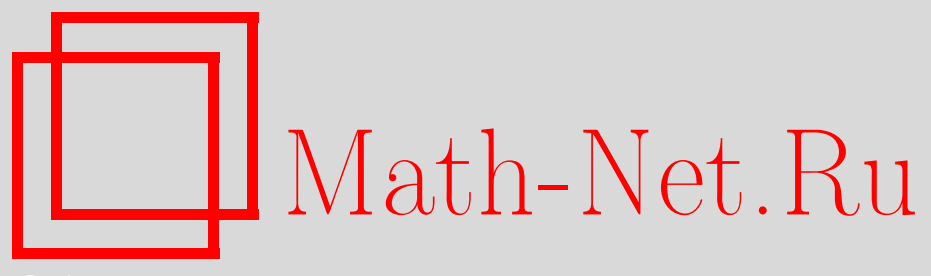

О. И. Мохов, О совместности детерминанта на кубических решетках, УМН, 2008, том 63, выпуск 6, 169-170

DOI: https://doi.org/10.4213/rm9239

Использование Общероссийского математического портала Math-Net.Ru подразумевает, что вы прочитали и согласны с пользовательским соглашением http://www . mathnet.ru/rus/agreement

Параметры загрузки:

IP: 54.237 .59 .107

26 апреля 2023 г., 15:16:30

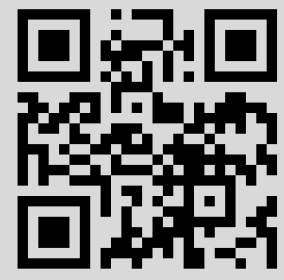




\section{О совместности детерминанта на кубических решетках}

\section{О. И. Мохов}

Рассмотрим квадратную решетку $\mathbb{Z}^{2}$ с узлами в точках с целочисленными координатами в $\mathbb{R}^{2}=\left\{\left(x_{1}, x_{2}\right) \mid x_{k} \in \mathbb{R}, k=1,2\right\}$ и комплексные (или вещественные) скалярные поля $u$ на решетке $\mathbb{Z}^{2}, u: \mathbb{Z}^{2} \rightarrow \mathbb{C}$, задаваемые своими значениями $u_{i_{1} i_{2}}$ $\left(u_{i_{1} i_{2}} \in \mathbb{C}\right)$ в каждом узле решетки с координатами $\left(i_{1}, i_{2}\right), i_{k} \in \mathbb{Z}, k=1,2$. Рассмотрим класс двумерных дискретных уравнений на решетке $\mathbb{Z}^{2}$ на поле $u$, задаваемых функциями четырех переменных $Q\left(x_{1}, x_{2}, x_{3}, x_{4}\right)$ с помощью уравнений

$$
Q\left(u_{i j}, u_{i+1, j}, u_{i, j+1}, u_{i+1, j+1}\right)=0, \quad i, j \in \mathbb{Z},
$$

так, что в каждом элементарном квадрате $2 \times 2$ решетки $\mathbb{Z}^{2}$, т. е. в узлах решетки с координатами вида $(i, j),(i+1, j),(i, j+1),(i+1, j+1), i, j \in \mathbb{Z}$, значение поля $u$ в одной из вершин квадрата определяется по значениям поля в трех других вершинах. В этом случае поле $u$ на решетке $\mathbb{Z}^{2}$ полностью определяется заданием начальных данных, например, на координатных осях решетки, $u_{i 0}$ и $u_{0 j}, i, j \in \mathbb{Z}$. В работах [1]-[3] интегрируемые дискретные уравнения вида (1) выделяются очень естественным условием совместности на кубических решетках (см. также [4]-[9]). Рассмотрим кубическую решетку $\mathbb{Z}^{3}$ с узлами в точках с целочисленными координатами в $\mathbb{R}^{3}=\left\{\left(x_{1}, x_{2}, x_{3}\right) \mid x_{k} \in \mathbb{R}, k=1,2,3\right\}$ и зададим начальные данные $u_{i 00}, u_{0 j 0}$ и $u_{00 k}, i, j, k \in \mathbb{Z}$, на координатных осях решетки. Двумерное дискретное уравнение (1) называется совместным на кубической решетке, если для начальных данных общего положения дискретное уравнение (1) может быть выполнено сразу на всех двумерных подрешетках кубической решетки $\mathbb{Z}^{3}$ (см. [1]-[5]). Классификации дискретных уравнений вида (1), совместных на кубической решетке, изучались в [4] и [8] при некоторых дополнительных условиях (см. также [9]). Примером такого двумерного нелинейного дискретного уравнения, совместного на кубической решетке, является уравнение, задаваемое детерминантом матрицы размером $2 \times 2$ значений поля в вершинах элементарного квадрата $2 \times 2$ решетки $\mathbb{Z}^{2}$ :

$$
u_{i, j+1} u_{i+1, j}-u_{i+1, j+1} u_{i j}=0, \quad i, j \in \mathbb{Z} .
$$

Уравнение (2) инвариантно относительно полной группы симметрий квадрата. Задание любых ненулевых начальных данных $u_{i 0}$ и $u_{0 j}, i, j \in \mathbb{Z}$, полностью определяет поле $u$ на решетке $\mathbb{Z}^{2}$, удовлетворяющее дискретному уравнению (2), а задание любых ненулевых начальных данных $u_{i 00}, u_{0 j 0}$ и $u_{00 k}, i, j, k \in \mathbb{Z}$, полностью определяет поле $u$ на решетке $\mathbb{Z}^{3}$, удовлетворяющее дискретному уравнению (2) на всех двумерных подрешетках кубической решетки $\mathbb{Z}^{3}$. Интегрируемость (в широком смысле) дискретного уравнения (2) очевидна, так как оно легко линеаризуется: $\log u_{i, j+1}+\log u_{i+1, j}-\log u_{i+1, j+1}-\log u_{i j}=0, i, j \in \mathbb{Z}$. В данной работе мы рассматриваем вопрос о совместности на кубической решетке для дискретных нелинейных уравнений, задаваемых детерминантами матриц более высокого порядка. Для них условие совместности на кубической решетке в том виде, как оно определено выше, не выполняется. Мы доказываем, что для них выполняется предлагаемое в данной работе другое, модифицированное условие совместности на кубической решетке. Рассмотрим дискретное уравнение на решетке $\mathbb{Z}^{2}$, задаваемое соотношением на значения поля $u$ в узлах решетки $\mathbb{Z}^{2}$, образующих элементарные квадраты $3 \times 3$ :

$$
Q\left(u_{i j}, \ldots, u_{i+s, j+r}, \ldots, u_{i+2, j+2}\right)=0, \quad 0 \leqslant s, r \leqslant 2, \quad i, j \in \mathbb{Z} .
$$

Работа выполнена при поддержке РФФИ (грант № 08-01-00054) и программы "Ведущие научные школы" (грант НШ-1824.2008.1). 
Задание начальных данных $u_{i 0}, u_{i 1}, u_{0 j}$ и $u_{1 j}, i, j \in \mathbb{Z}$, полностью определяет поле $u$ на решетке $\mathbb{Z}^{2}$, удовлетворяющее дискретному уравнению (3). Рассмотрим кубическую решетку $\mathbb{Z}^{3}$ и условие совместности дискретного уравнения (3) на всех двумерных подрешетках кубической решетки $\mathbb{Z}^{3}$. Начальные данные могут быть заданы, например, в следующих узлах решетки: $u_{i 00}, u_{i 10}, u_{i 01}, u_{i 11}, u_{0 j 0}, u_{1 j 0}, u_{0 j 1}, u_{1 j 1}$, $u_{00 k}, u_{10 k}, u_{01 k}$ и $u_{11 k}, i, j, k \in \mathbb{Z}$. В кубе $\{(i, j, k) \mid 0 \leqslant i, j, k \leqslant 2\}$ значения $u_{202}$, $u_{212}, u_{220}, u_{221}, u_{022}$ и $u_{122}$ определяются соотношениями (3), а для значения $u_{222}$ должны выполняться три соотношения на трех гранях куба. Рассмотрим дискретное нелинейное уравнение на решетке $\mathbb{Z}^{2}$, задаваемое детерминантом матрицы значений поля $u$ в узлах решетки $\mathbb{Z}^{2}$, образующих элементарные квадраты $3 \times 3$ :

$$
\begin{aligned}
& u_{i, j+2} u_{i+1, j+1} u_{i+2, j}+u_{i, j+1} u_{i+1, j} u_{i+2, j+2}+u_{i, j} u_{i+1, j+2} u_{i+2, j+1}-u_{i, j} u_{i+1, j+1} u_{i+2, j+2} \\
& -u_{i, j+2} u_{i+1, j} u_{i+2, j+1}-u_{i, j+1} u_{i+1, j+2} u_{i+2, j}=0, \quad i, j \in \mathbb{Z} .
\end{aligned}
$$

Уравнение (4) инвариантно относительно полной группы симметрий конфигурации узлов решетки $\mathbb{Z}^{2}$, образующей элементарные квадраты $3 \times 3$. Для начальных данных общего положения дискретное уравнение (4) не является совместным на двумерных подрешетках кубической решетки $\mathbb{Z}^{3}$. Рассмотрим другое, модифицированное условие совместности на кубической решетке для дискретного нелинейного уравнения (4). Мы потребуем, чтобы дискретное уравнение (4) было выполнено не только на всех двумерных подрешетках кубической решетки $\mathbb{Z}^{3}$, но и на всех объединениях двух любых пересекающихся двумерных подрешеток кубической решетки $\mathbb{Z}^{3}$, т. е. соответствующие элементарные квадраты $3 \times 3$, на которых рассматривается дискретное уравнение (4), могут быть изогнуты под прямым углом, переходя с одной двумерной подрешетки на другую, например, $\{(i, 0,0),(i, 1,0),(i, 0,1), i=0,1,2\}$, $\{(0, j, 0),(1, j, 0),(0, j, 1), j=0,1,2\}$ и $\{(0,0, k),(1,0, k),(0,1, k), k=0,1,2\}$. В этом случае начальные данные могут быть заданы, например, в следующих узлах кубической решетки $\mathbb{Z}^{3}: u_{i 00}, u_{i 01}, u_{2 j 0}, u_{2 j 1}, u_{10 k}, u_{20 k}, u_{110}$ и $u_{112}, i, j, k \in \mathbb{Z}$. Соответствующие дискретные уравнения мы будем также называть совместными на кубической решетке. Тогда имеет место следующая теорема.

Теорема 1. Для любых началъных данных общего положения нелинейное дискретное уравнение (4) может быть выполнено совместным образом на всех объединениях двух любых двумерных подрешеток кубической решетки $\mathbb{Z}^{3}$, m. е. дискретное нелинейное уравнение (4) является совместным на кубической решетке $\mathbb{Z}^{3}$.

Аналогичное свойство совместности на кубической решетке имеет место для детерминанта любого порядка $N$.

Теорема 2. Для любого заданного натурального числа $N$ дискретное нелинейное уравнение, задаваемое детерминантом матрицы значений поля и в узлах решет$\kappa и \mathbb{Z}^{2}$, образующих элементарные квадраты $N \times N$, является совместным на кубической решетке $\mathbb{Z}^{3}$.

\section{Список литературы}

[1] F. W. Nijhoff, A. J. Walker, Glasg. Math. J., 43A (2001), 109-123. [2] F. W. Nijhoff, Phys. Lett. A, 297:1-2 (2002), 49-58. [3] A. I. Bobenko, Yu. B. Suris, Int. Math. Res. Not., 11 (2002), 573-611. [4] V. E. Adler, A. I. Bobenko, Yu. B. Suris, Comm. Math. Phys., 233:3 (2003), 513-543. [5] А. I. Bobenko, Yu. B. Suris, arXiv: math/0504358. [6] А. И. Бобенко, Ю. Б. Сурис, УМН, 62:1 (2007), 3-50. [7] А. П. Веселов, УМH, 46:5 (1991), 3-45. [8] V. E. Adler, A. I. Bobenko, Yu. B. Suris, arXiv: math/ 0705.1663. [9] S. P. Tsarev, Th. Wolf, Lett. Math. Phys., 84:1 (2008), $31-39$.

О. И. Мохов (O. I. Mokhov)

Центр нелинейных исследований при Институте теоретической физики им. Л. Д. Ландау РАН

E-mail: mokhov@mi.ras.ru, mokhov@landau.ac.ru
Представлено С. П. Новиковым Принято редколлегией 06.08.2008 